\title{
Robust Speed-sensorless Vector Control of Doubly Fed Induction Motor Drive Using Sliding Mode Rotor Flux Observer
}

\author{
Djamila Cherifi, Yahia Miloud \\ Faculty of Technology, Department of Electrical Engineering, Dr. Moulay Tahar University, Algeria
}

\begin{tabular}{l}
\hline \hline Article Info \\
\hline Article history: \\
Received Jun 15, 2018 \\
Revised Aug 20, 2018 \\
Accepted Sep 8, 2018 \\
\hline Keyword: \\
Double-Fed Induction Machine \\
Field oriented control \\
Robust control \\
Sensorless control \\
Sliding mode observer \\
\end{tabular}

\begin{abstract}
This paper presents a robust observer for sensorless speed control of Doubly Fed Induction Motor (DFIM), based on the slidin mode. In the first step, a model of the doubly fed induction motor fed by two PWM inverters with separate DC bus link is developed. In the second step and in order to provide a robust separate control between flux and motor speed a vector control by field oriented strategy applying a sliding mode regulator was implemented. Finally, speed estimation of a doubly fed induction motor based on sliding mode observer is presented. The simulation tests schow the effectiveness of the proposed method especially in the load disturbances, the change of the refrence speed and low speed. Also the influence of parameter variations will be studied by simulation.
\end{abstract}

Copyright (C) 2018 Institute of Advanced Engineering and Science. All rights reserved.

\section{Corresponding Author:}

Djamila Cherifi,

Faculty of Technology,

Department of Electrical Engineering,

Dr. Moulay Tahar University, Algeria.

Email: d_cherifi@yahoo.fr

\section{INTRODUCTION}

Nowadays, several works have been directed towards the study of the double-feed induction machine (DFIM), due to its several advantages as well as motor application in high power applications such as traction, marine propulsion or as generator in wind energy conversion systems like wind turbine, or pumped storage systems [1-2].

The DFIM has some distinct advantages compared to the conventional squirrel-cage machine. The DFIM can be fed and controlled stator or rotor by various possible combinations. Indeed, the inputcommands are done by means of four precise degrees of control freedom relatively to the squirrel cage induction machine where its control appears quite simpler [3-4].

However, these advantages have long been inhibited by the complexity of the control, [5]. In order to obtain an DFIM having similar performance to a DC machine where there is a natural decoupling between the magnitude controlling the flux (the excitation current) and the magnitude related to the torque (the armature current) [6], several methods are used. Such as the vector control (field oriented control) which gives the decoupling between the torque and the flux like DC motor, [7].

The control laws using the PID type regulators give good results in the case of linear systems with constant parameters, but for nonlinear systems, these conventional control laws may be insufficient because they are not robust especially when the requirements on the speed and other dynamic characteristics of the system are strict. Therefor robust control laws must be used, such as sliding mode speed controller wich is insensitive to parameter variations, disturbances, and nonlinearities, [8]. 
But, the knowledge of the rotor speed is necessary, this necessity requires additional speed sensor which adds to the cost and the complexity of the drive system. Over the past few years, ongoing research has concentrated on the elimination of the speed sensor at the machine shaft without deteriorating the dynamic performance of the drive control system. The advantages of speed sensorless DFIM drives are reduced hardware complexity and lower cost, reduces size of the drive machine, elimination of the sensor cable, better noise immunity, increased reliability and less maintenance requirements, [5], [9].

In order to achieve good performance of sensorless vector control, different speed estimation schemes have been proposed, and a variety of speed estimators exist nowdays [10], such as direct calculation method, model reference adaptive system (MRAS), Extended Kalman Filters (EKF), Extended Luenberger observer (ELO), sliding mode observer ect, [11-12].

Among various approaches, sliding mode observer based speed sensorless estimation has been recently used, due to its good performance and case of implementation. The sliding mode (SMO) belongs to the group of closed loop observers. It is a deterministic type of observer because it is based on a deterministic model of the system [13].

This paper is organized as follows: section 2 dynamic model of DFIM is reported; principle of fieldoriented controller is given in section 3. The proposed solution is presented in section 4 . In section 5, results of simulation tests are reported. Finally, section 6 draws conclusions.

\section{DOUBLY FED INDUCTION MODEL}

The chain of energy conversion adopted for the power supply of the DFIM consists of two converters, one on the stator and the other one on the rotor. A filter is inserted between the two converters, as shown in Figure 1.

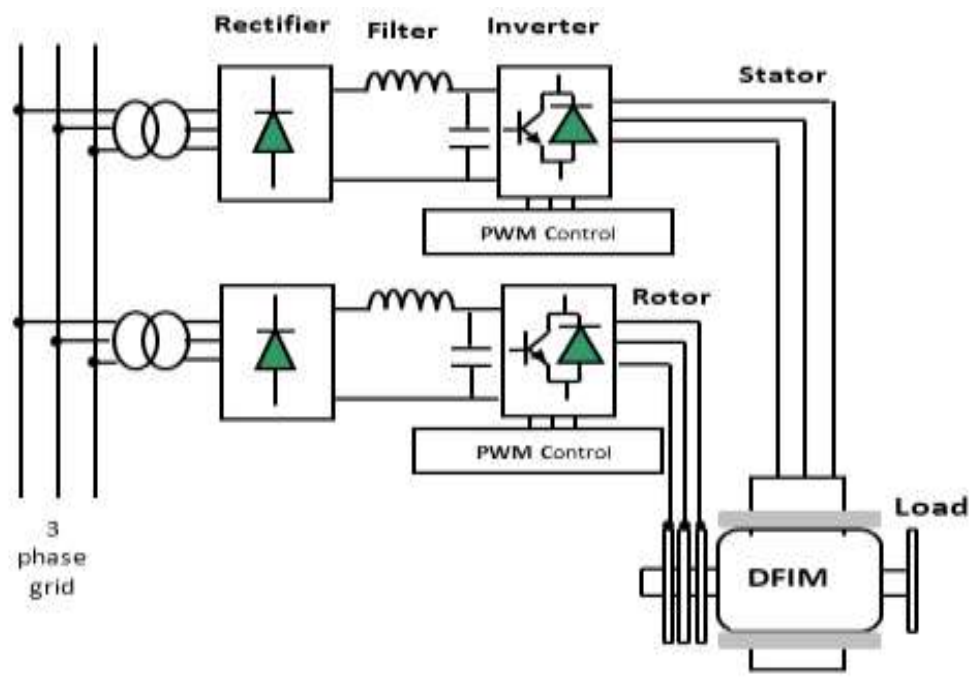

Figure 1. General scheme of DFIM drive installation

The structure of DFIM is very complex. Therefore, in order to develop a model, it is necessary to consider the following simplifying assumptions: the machine is symmetrical with constant air gap; the magnetic circuit is not saturated and it is perfectly laminated, with the result that the iron losses and hysteresis are negligible and only the windings are driven by currents; the f.m.m created in one phase of stator and rotor are sinusoidal distributions along the gap [14]. By this means, a dynamic model of the doubly fed induction motor in stationary reference frame can be expressed by: 


$$
\left\{\begin{array}{l}
\frac{d}{d t} i_{s d}=-\lambda i_{s d}+\omega_{s} i_{s q}+\frac{K}{T_{r}} \phi_{r d}+\omega \cdot K \phi_{r q}+\frac{1}{\sigma L_{s}} v_{s d}-K v_{r d} \\
\frac{d}{d t} i_{s q}=-\omega_{s} i_{s q}-\lambda i_{s q}-\omega \cdot K \phi_{r d}+\frac{K}{T_{r}} \phi_{r q}+\frac{1}{\sigma L_{s}} v_{s q}-K v_{r q} \\
\frac{d}{d t} \phi_{r d}=\frac{L_{m}}{T_{r}} i_{s d}-\frac{1}{T_{r}} \phi_{r d}+\omega . \phi_{q r}+v_{r d} \\
\frac{d}{d t} \phi_{r q}=\frac{L_{m}}{T_{r}} i_{s q}-\omega \cdot \phi_{r d}-\frac{1}{T_{r}} \phi_{r q}+v_{r q} \\
\frac{d}{d t} \omega=p^{2} \frac{L_{m}}{L_{r}}\left(\phi_{r d} i_{s q}-\phi_{r q} i_{s d}\right)-\frac{f}{J} \omega-\frac{C_{r}}{J}
\end{array}\right.
$$

with:

$$
T_{r}=\frac{L_{r}}{R_{r}} ; T_{s}=\frac{L_{s}}{R_{s}} ; \lambda=\frac{1}{\sigma . T_{r}} ; K=\frac{L_{m}}{\sigma L_{s} L_{r}} ; \quad \sigma=1-\frac{L_{m}^{2}}{L_{s} L_{r}} ; \quad \omega=p . \Omega
$$

The electromagnetic torque is expressed by:

$$
T_{e m}=\frac{p L_{m}}{L_{r}}\left(\phi_{r d} \cdot i_{s q}-\phi_{r q} \cdot i_{s d}\right)
$$

\section{VECTOR CONTROL BY DIRECT ROTOR FLUX ORIENTATION}

The main objective of the vector control of DFIM is as in DC machines, to independently control the torque and the flux; this is done by using a $d$ - $q$ rotating reference frame synchronously with the rotor flux space vector. The d-axis is then aligned with the rotor flux space vector [6]. Under this condition we get:

$$
\phi_{r q}=0, \phi_{r}=\phi_{r d}
$$

Figure 2 shows the structure for the rotor field orientation on the $d$-axis.

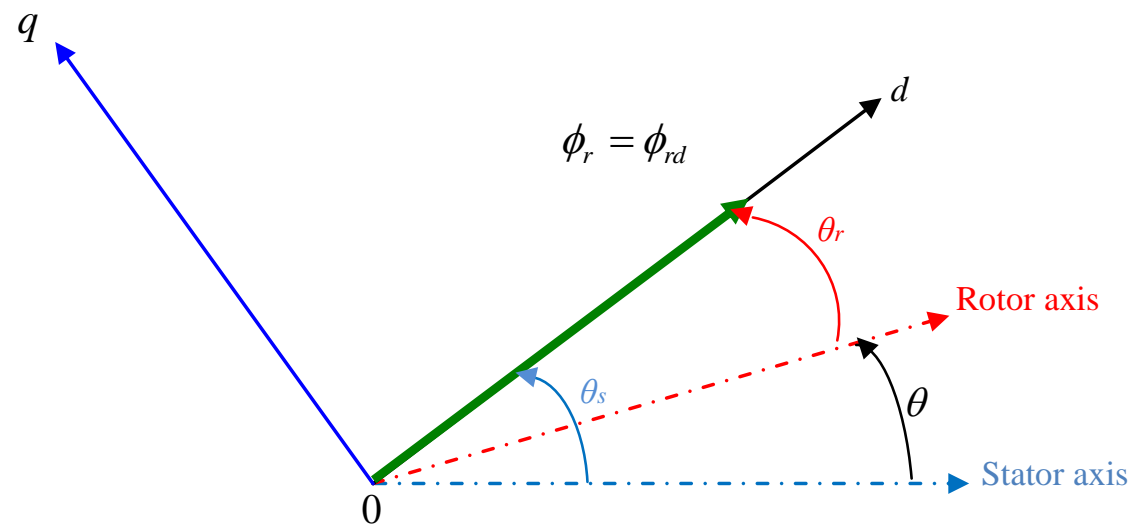

Figure 2. Rotor field orientation on the d-axis

So, we can write :

$$
T_{e m}=\frac{p L_{m}}{L_{r}}\left(\phi_{r d} . i_{s q}\right)
$$


For the direct rotor flux orientation (DFOC) of DFIM, accurate knowledge of the magnitude and position of the rotor flux vector is necessary. In a DFIM motor mode, as stator and rotor currents are measurable, the rotor flux can be estimated (calculated). The flux estimator can be obtained by the following equations, [15]:

$$
\phi_{r}=\sqrt{\phi_{r \alpha}^{2}+\varphi_{r \beta}{ }^{2}} \text { and } \theta_{s}=\tan ^{-1}\left(\frac{\phi_{r \beta}}{\phi_{r \alpha}}\right)
$$

\subsection{Sliding mode speed control}

A Sliding Mode Controller (SMC) is a Variable Structure Controller (VSC). SMC method is a kind of robust control technique which is extensively utilized in nonlinear systems where parameter uncertainties exist. Basically, a VSC includes several different continuous functions that can map plant state to a control surface, whereas switching among different functions is determined by plant state represented by a switching function [16]. The design of the control system will be demonstrated for a following nonlinear system, [17]:

$$
\dot{x}=f(x, t)+B(x, t) \cdot u(x, t)
$$

where:

$$
\begin{aligned}
& x \in \mathfrak{R}^{n} \text { is the state vector } \\
& u \in \mathfrak{R}^{m} \text { is the control vector } \\
& f(x, t) \in \mathfrak{R}^{n \times m}
\end{aligned}
$$

The control law satisfies the precedent conditions is presented in the following form:

$$
u=u^{e q}+u^{n}, u^{n}=-k_{f} \operatorname{sgn}(S(x))
$$

where $u$ is the control vector, $u^{e q}$ is the equivalent control vector, $u^{n}$ is the switching part of the control (the correction factor), $k_{f}$ is the controller gain. $u^{e q}$ can be obtained by considering the condition for the sliding regimen, $S(x)=0$.

The equivalent control keeps the state variable on sliding surface, once they reach it.

For a defined function, [18]:

$$
\operatorname{sgn}(S(x))=\left\{\begin{array}{lll}
1, & \text { if } & S(x)>0 \\
0, & \text { if } & S(x)=0 \\
-1, & \text { if } & S(x)<0
\end{array}\right.
$$

\subsubsection{Speed control}

Speed adjustment is done by controlling the stator current $I_{s q}$.

So, the command law can be expressed as:

$$
I_{s q}^{r e f}=I_{s q}^{e q}+I_{s q}^{n}
$$

The expression of the speed control surface has the form:

$$
S(\omega)=\omega_{r e f}-\omega
$$

The derivative of the surface is

$$
\dot{S}(\omega)=\dot{\omega}_{r e f}-\dot{\omega}
$$

With the mechanical equation equal to: 


$$
\dot{\omega}=\frac{P . L_{m}}{J . L_{r}}\left(I_{s q} \cdot \phi_{r d}^{r e f}\right)-\frac{p}{J} C_{r}-\frac{f}{J} \omega
$$

By replacing the mechanical equation in the equation of the switching surface, the derivative of the surface becomes:

$$
\dot{S}(\omega)=\dot{\omega}_{r e f}-\left(\frac{P . L_{m}}{J \cdot L_{r}}\left(I_{s q} \cdot \phi_{r d}^{r e f}\right)-\frac{p}{J} C_{r}-\frac{f}{J} \omega\right)
$$

By replacing the current $I_{s q}$ by the current $I_{s q}^{r e f}=I_{s q}^{e q}+I_{s q}^{n}$, it is found that the command appears explicitly in the derivative of the surface, the latter will be written in the following form:

$$
\dot{S}(\omega)=\dot{\omega}_{r e f}-\left(\frac{P . L_{m} \cdot \phi_{r d}^{r e f}}{J \cdot L_{r}} I_{s q}^{e q}+\frac{P \cdot L_{m} \cdot \phi_{r d}^{r e f}}{J \cdot L_{r}} I_{s q}^{n}-\frac{p}{J} C_{r}-\frac{f}{J} \omega\right)
$$

During the slip mode and in steady state, we have:

$$
S(\omega)=0, \dot{S}(\omega)=0, I_{s q}^{n}=0
$$

From which one derives the magnitude of equivalent command, $I_{s q}^{e q}$ is written:

$$
I_{s q}^{e q}=\frac{J \cdot L_{r}}{P . L_{m} \cdot \phi_{r d}^{r e f}}\left(\dot{\omega}_{r e f}+\frac{P}{J} C_{r}+\frac{f}{J} \omega\right)
$$

During the convergence mode, condition $\dot{V}(\omega)=S(\omega) \cdot \dot{S}(\omega)<0$ must be verified. By replacing the expression of the equivalent command in the expression of the derivative of the surface, we obtain:

$$
\dot{S}(\omega)=-\frac{P \cdot L_{m} \cdot \phi_{r d}^{r e f}}{J \cdot L_{r}} I_{s q}^{n}
$$

In which $I_{s q}^{n}=K_{i_{s q}} \operatorname{sign}(S(\omega))$

To check the stability condition of the system, the $K_{i_{s q}}$ constant must be positive.

\section{SLIDING MODES OBSERVER}

Here, a sliding mode observer is studied for the estimation of the speed of rotation of the DFIM, this observer used the measurements of the stator voltage and current, [19].

Based on the equations of the stator currents and the equations of the rotor flux of the machine in the fixed reference frame $(\alpha, \beta)$, we can write, $[20]$ :

$$
\left\{\begin{array}{l}
\frac{d}{d t} i_{s \alpha}=-\lambda i_{s \alpha}+\omega_{s} i_{s \beta}+\frac{K}{T_{r}} \phi_{r \alpha}+\omega \cdot K \phi_{r \beta}+\frac{1}{\sigma L_{s}} v_{s \alpha}-K v_{r \alpha} \\
\frac{d}{d t} i_{s \beta}=-\omega_{s} i_{s \beta}-\lambda i_{s \beta}-\omega \cdot K \phi_{r \alpha}+\frac{K}{T_{r}} \phi_{r \beta}+\frac{1}{\sigma L_{s}} v_{s \beta}-K v_{r \beta} \\
\frac{d}{d t} \phi_{r \alpha}=\frac{L_{m}}{T_{r}} i_{s \alpha}-\frac{1}{T_{r}} \phi_{r \alpha}+\omega \cdot \phi_{r \beta}+v_{r \alpha} \\
\frac{d}{d t} \phi_{r \beta}=\frac{L_{m}}{T_{r}} i_{s \beta}-\omega \cdot \phi_{r \alpha}-\frac{1}{T_{r}} \phi_{r \beta}+v_{r \beta}
\end{array}\right.
$$


with:

$$
T_{r}=\frac{L_{r}}{R_{r}} ; T_{s}=\frac{L_{s}}{R_{s}} ; \lambda=\frac{1}{\sigma . T_{r}} ; K=\frac{L_{m}}{\sigma L_{s} L_{r}} ; \quad \sigma=1-\frac{L_{m}^{2}}{L_{s} L_{r}}
$$

A sliding mode observer is an observer whose gain-correcting term contains the discontinuous function: sign. The sliding modes are control techniques based on the theory of systems with variable structure, [17].

The dynamics of observers by sliding modes concerning the observation error of state $e=x-\hat{x}$. Their evolution is imposed on a variety of surfaces, on which the error of estimating the output $e=y-\hat{y}$ tending towards zero, [18].

Thus, the dynamics on this surface variety will be stabilized, or assigned, so as to limit or cancel the estimation error. However, a sliding mode observer is written in the form:

$$
\left\{\begin{array}{l}
\dot{\hat{x}}=f(\hat{x}, u)+G_{g} \operatorname{sign}(y-\hat{y}) \\
\hat{y}=h(\hat{x})
\end{array}\right.
$$

With, $\hat{x}$ : Estimated state

$u$ : Observer input or command $y$ and $\hat{y}$ : Measured and estimated outputs, respectively.

Or $\operatorname{sign}(y-\hat{y})=\left[\operatorname{sign}\left(y_{1}-\hat{y}_{1}\right) \quad \operatorname{sign}\left(y_{2}-\hat{y}_{2}\right) \ldots \ldots . . . \operatorname{sign}\left(y_{p}-\hat{y}_{p}\right)\right] G_{g}:$ Matrix observer gain

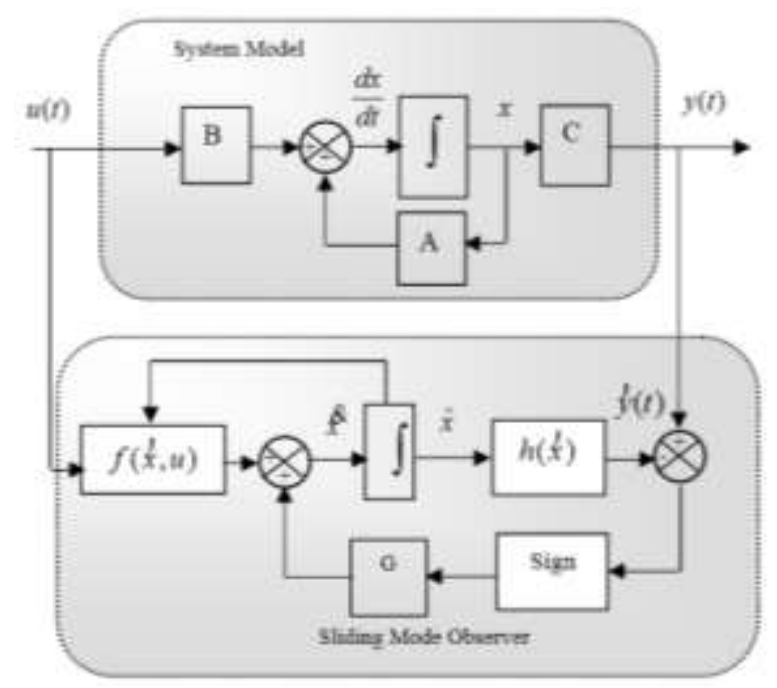

Figure 3. State space form of an sliding mode observer

Let us, $\hat{x}_{1}, \hat{x}_{2}, \hat{x}_{3}, \hat{x}_{4}$ the estimates of the $x_{1}, x_{2}, x_{3}, x_{4}$ respectively which are the state variables of $i_{s \alpha}, i_{s \beta}, \phi_{r \alpha}, \phi_{r \beta}$. The observer is only a copy of the original system to which one adds the control gains with the terms of commutation; thus, [19]: 


$$
\left\{\begin{array}{l}
\dot{\hat{x}}_{1}=-\lambda \hat{x}_{1}+\omega_{s} \hat{x}_{2}+\frac{K}{T_{r}} \hat{x}_{3}+\omega \cdot K \hat{x}_{4}+\frac{1}{\sigma L_{s}} v_{s \alpha}-K v_{r \alpha}+g_{1} I_{s} \\
\dot{\hat{x}}_{2}=-\omega_{s} \hat{x}_{2}-\lambda \hat{x}_{2}-\omega \cdot K \hat{x}_{3}+\frac{K}{T_{r}} \hat{x}_{4}+\frac{1}{\sigma L_{s}} v_{s \beta}-K v_{r \beta}+g_{2} I_{s} \\
\dot{\hat{x}}_{3}=\frac{L_{m}}{T_{r}} \hat{x}_{1}-\frac{1}{T_{r}} \hat{x}_{3}+\omega \cdot \hat{x}_{4}+v_{r \alpha}+g_{3} I_{s} \\
\dot{\hat{x}}_{4}=\frac{L_{m}}{T_{r}} \hat{x}_{2}-\omega \cdot \hat{x}_{3}-\frac{1}{T_{r}} \hat{x}_{4}+v_{r \beta}+g_{4} I_{s}
\end{array}\right.
$$

where $g_{1}, g_{2}, g_{3}, g_{4}$ are observer gains, $g_{j}=\left[\begin{array}{ll}g_{j 1} & g_{j 2}\end{array}\right]$ for $j \in\{1,2,3,4\}$

The vector $I_{s}$ is given by:

$$
I_{s}=\left[\begin{array}{l}
\operatorname{sign}\left(S_{1}\right) \\
\operatorname{sign}\left(S_{2}\right)
\end{array}\right]
$$

With $\quad S_{o b}=\left[\begin{array}{l}S_{1} \\ S_{2}\end{array}\right]=\Gamma\left[\begin{array}{l}x_{1}-\hat{x}_{1} \\ x_{2}-\hat{x}_{2}\end{array}\right]=\Gamma\left[\begin{array}{l}i_{s \alpha}-\hat{i}_{s \alpha} \\ i_{s \beta}-\hat{i}_{s \beta}\end{array}\right]$

And

$$
\Gamma=\frac{1}{\beta(t)}\left[\begin{array}{cc}
\frac{K}{T_{r}} & -\omega(t) K \\
\omega(t) K & \frac{K}{T_{r}}
\end{array}\right]
$$

With

$$
\beta(t)=\left[\frac{K}{T_{r}}\right]^{2}+\omega^{2}(t) K^{2}
$$

The choice of $\Gamma$ is made in order to facilitate the calculation of gains observer. Let $e_{i}=x_{j}-\hat{x}_{j}$ for $\mathrm{j} \in\{1,2,3,4\}$ the dynamics of estimation error are given by:

$$
\begin{aligned}
& \dot{e}_{1}=\frac{K}{T_{r}} e_{3}+\omega K e_{4}-g_{1} I_{s} \\
& \dot{e}_{2}=\frac{K}{T_{r}} e_{4}-\omega K e_{3}-g_{2} I_{s} \\
& \dot{e}_{3}=-\frac{1}{T_{r}} e_{3}-\omega e_{4}-g_{3} I_{s} \\
& \dot{e}_{4}=-\frac{1}{T_{r}} e_{4}+\omega e_{3}-g_{4} I_{s}
\end{aligned}
$$

The analysis of stability consists in determining the gains $g_{1}$ and $g_{2}$ in order to ensure the attractivity of the sliding surface $\mathrm{S}_{\mathrm{ob}}=0$. Then $g_{3}$ and $g_{4}$ are given such as the reduced system obtained when $\mathrm{S}_{\mathrm{ob}} \equiv \dot{\mathrm{S}} \equiv 0$ is locally stable. The following result is obtained.

Let us suppose that the state variables $\mathrm{x}_{3}(\mathrm{t})$ and $\mathrm{x}_{4}(\mathrm{t})$ are boundeds and let us consider the system (23) with the following gains: 


$$
\begin{aligned}
& {\left[\begin{array}{ll}
g_{1} & g_{2}
\end{array}\right]=\left[\begin{array}{ll}
g_{11} & g_{12} \\
g_{21} & g_{22}
\end{array}\right]=\Gamma^{-1} \Delta, \quad \Delta=\left[\begin{array}{cc}
\delta_{1} & 0 \\
0 & \delta_{2}
\end{array}\right]} \\
& {\left[\begin{array}{ll}
g_{31} & g_{32} \\
g_{41} & g_{42}
\end{array}\right]=\left[\begin{array}{cc}
\left(q_{1}-\frac{1}{T_{r}}\right) \delta_{1} & -\omega(t) \delta_{2} \\
\omega(t) \delta_{1} & \left(q_{2}-\frac{1}{T_{r}}\right) \delta_{2}
\end{array}\right]}
\end{aligned}
$$

where $\left\{\begin{array}{l}\delta_{1}>\rho_{3}+\left|\hat{\phi}_{r \alpha}\right|+a_{\max }\left|e_{1}\right|+b_{\text {max }}\left|e_{2}\right| \\ \delta_{2}>\rho_{4}+\left|\hat{\phi}_{r \beta}\right|+b_{\text {max }}\left|e_{1}\right|+a_{\text {max }}\left|e_{2}\right|\end{array}\right.$

With: $a_{\max }=2 T_{r} p^{2} K \eta_{1} \eta_{2}, b_{\max }=p T_{r}^{2} \eta_{2}\left(\frac{1}{K}+2 p^{2} \eta_{1}^{2}\right)$

$$
\left|x_{3}(t)\right| \leq \rho_{3},\left|x_{4}(t)\right| \leq \rho_{4}, q_{1} q_{2}>0
$$

The variety with two dimensions $\mathrm{S}_{\mathrm{ob}}=0$ is attractive and $e_{1}(\mathrm{t}), e_{2}(\mathrm{t})$ converges towards zero.

The dynamics of a reduced order obtained when $\mathrm{S}_{\mathrm{ob}} \equiv \dot{\mathrm{S}} \equiv 0$ is given by:

$$
\left\{\begin{array}{l}
\dot{e}_{3}=-q_{1} e_{3} \\
\dot{e}_{4}=-q_{2} e_{4}
\end{array}\right.
$$

Where $\mathrm{q}_{1}, \mathrm{q}_{2}>0$ that corresponds to an exponential stability of $\mathrm{e}_{3}$ and $\mathrm{e}_{4}$.

\subsection{Estimation of speed by sliding mode}

Consider the error dynamics of the flux observer given by Equation (24), this equation can be rewritten in the following form, [17]:

$$
\dot{e}(\omega)=A(\omega) \cdot e+C_{g}(\omega) \cdot I_{s g}(\omega)
$$

With

$$
\dot{e}(\omega)=\left[\begin{array}{c}
\dot{e}_{1} \\
\dot{e}_{2} \\
\dot{e}_{3} \\
\dot{e}_{4}
\end{array}\right] ; A(\omega)=\left[\begin{array}{cccc}
0 & 0 & \frac{K}{T_{r}} & K . \omega \\
0 & 0 & -K . \omega & \frac{K}{T_{r}} \\
0 & 0 & \frac{-1}{T_{r}} & -\omega \\
0 & 0 & \omega & \frac{-1}{T_{r}}
\end{array}\right] ; G_{g}(\omega)=\left[\begin{array}{cc}
\frac{K}{T_{r}} \cdot \delta_{1} & \frac{K}{T_{r}} \cdot \omega \cdot \delta_{2} \\
-\frac{K}{T_{r}} \cdot \omega \cdot \delta_{1} & \frac{K}{T_{r}} \cdot \delta_{2} \\
\left(q_{1}-\frac{1}{T_{r}}\right) \cdot \delta_{1} & -\omega \cdot \delta_{2} \\
\omega . \delta_{1} & \left(q_{2}-\frac{1}{T_{r}}\right) \cdot \delta_{2}
\end{array}\right]
$$

Suppose now that the rotor speed $\omega$ is replaced by its estimated $\hat{\omega}=\omega-\Delta \omega$, the Equation (25) becomes:

$$
\dot{e}(\hat{\omega})=A(\hat{\omega}) \cdot e+G_{g}(\hat{\omega}) \cdot I_{s g}(\hat{\omega})
$$

With

$$
A(\hat{\omega})=A(\omega)+\Delta \Omega
$$




$$
\begin{aligned}
& G_{g}(\hat{\omega})=G_{g}(\omega)+\Delta G_{g} \\
& I_{s g}=\operatorname{sign}\left[\begin{array}{c}
S_{1}+\frac{K}{T_{r}} \cdot e_{2} \cdot \Delta \omega \\
\beta \\
S_{2}+\frac{\frac{K}{T_{r}} \cdot e_{1} \cdot \Delta \omega}{\beta}
\end{array}\right]
\end{aligned}
$$

And

$$
\Delta A=\left[\begin{array}{cccc}
0 & 0 & 0 & -K . \Delta \omega \\
0 & 0 & K . \Delta \omega & 0 \\
0 & 0 & 0 & \Delta \omega \\
0 & 0 & -\Delta \omega & 0
\end{array}\right] ; \Delta G_{g}=\left[\begin{array}{cc}
0 & \frac{K}{T_{r}} \cdot \Delta \omega \cdot \delta_{2} \\
-\frac{K}{T_{r}} . \Delta \omega \cdot \delta_{1} & 0 \\
0 & \Delta \omega . \delta_{2} \\
-\Delta \omega . \delta_{1} & 0
\end{array}\right]
$$

The idea is to apply the criterion of stability of lyapunov to see the convergence of the error towards zero, for this one chooses the function of lyapunov of the following form [19]:

$$
v=\frac{1}{2} e \cdot e^{T}+\frac{1}{2 \lambda}(\Delta \omega)^{2}
$$

The derivative of Equation (30) with respect to time is:

$$
\dot{v}=e^{T} . \dot{e}(\hat{\omega})+\frac{1}{\lambda} \Delta \omega \cdot \dot{\hat{\omega}} \dot{v}=e^{T} \cdot \dot{e}(\hat{\omega})+\frac{1}{\lambda} \Delta \omega \cdot \dot{\hat{\omega}}
$$

Let us replace $\dot{e}(\hat{\omega})$ by its value, then Equation (31) becomes:

$$
\dot{v}=e^{T}\left\{(A(\omega)+\Delta A) \cdot e-\left(G_{g}+\Delta G_{g}\right) \cdot I_{s g}(\omega)\right\}+e^{T} \cdot G_{g} \cdot I_{s g}-e^{T} \cdot G_{g} \cdot I_{s g}+\frac{1}{\lambda} \Delta \omega \cdot \dot{\hat{\omega}}
$$

Finally we will have:

$$
\dot{v}=e^{T}\left[\left(A(\omega) e-G_{g} \cdot I_{s g}(\omega)\right)+\left(G_{g} \cdot I_{s g}(\omega)-\left(G_{g}+\Delta G_{g}\right) \cdot I_{s g}(\omega)\right)\right]+\frac{1}{\lambda} \Delta \omega \cdot \dot{\hat{\omega}}+e^{T} . \Delta A \cdot e
$$

With

$$
e^{T} . \Delta A \cdot e=\Delta \omega \cdot\left\{p \cdot K \cdot\left(e_{1} \cdot \hat{x}_{4}-e_{2} \cdot \hat{x}_{3}\right)\right\}+p \cdot K \cdot \Delta \omega\left(e_{2} \cdot x_{3}-e_{1} \cdot x_{4}\right)
$$

we do the following equality :

$$
\frac{1}{\lambda} \Delta \omega \cdot \dot{\hat{\omega}}+\Delta \omega \cdot\left\{p \cdot K \cdot\left(e_{1} \cdot \hat{x}_{4}-e_{2} \cdot \hat{x}_{3}\right)\right\}=0
$$

From Equation (35) and if $\Delta \omega \neq 0$, an adaptation law for the rotor speed is deduced:

$$
\begin{aligned}
& \dot{\hat{\omega}}=\lambda \cdot K \cdot p \cdot\left(e_{1} \cdot \hat{x}_{4}-e_{2} \cdot \hat{x}_{3}\right) \\
& \dot{\hat{\omega}}=\lambda \cdot K \cdot p \cdot\left(\left(i_{s \alpha}-\hat{i}_{s \alpha}\right) \hat{\phi}_{r \beta}-\left(i_{s \beta}-\hat{i}_{s \beta}\right) \cdot \hat{\phi}_{r \alpha}\right)
\end{aligned}
$$


Equation (33) then becomes:

$$
\dot{v}=e^{T} . e+e^{T} \cdot\left\{G_{g} \cdot I_{s g}(\hat{\omega})-\left(G_{g}+\Delta G_{g}\right) \cdot I_{s g}(\hat{\omega})\right\}+p \cdot K \cdot \Delta \omega \cdot\left(e_{2} \cdot x_{3}-e_{1} \cdot x_{4}\right)
$$

The term $e^{T} . e$ being defined negative by the sliding modes, therefore, the system is globally stable if and only if the following equation is satisfied:

$$
e^{T} \cdot\left\{G_{g} \cdot I_{s g}(\hat{\omega})-\left(G_{g}+\Delta G_{g}\right) \cdot I_{s g}(\hat{\omega})\right\}+p \cdot K . \Delta \omega \cdot\left(e_{2} \cdot x_{3}-e_{1} \cdot x_{4}\right)<0
$$

This equation represents the domain of stability, so it is enough to respect this condition so that the errors ' $e$ ' and $\Delta \omega$ converge asymptotically towards zero.

\section{SIMULATION RESULTS AND DISCUSSION}

To demonstrate the faisability of the proposed estimation algorithm, and incorporated into a speed control system of a DFIM with direct oriented rotor flux, the simulation of the complete system, figure 4 was carried out using different of cases that wil be presented and discussed next. The first test concerns a no-load starting of the motor with a reference speed $\boldsymbol{\Omega}_{\mathrm{ref}=} 150 \mathrm{rad} / \mathrm{s}$. and a nominal load disturbance torque (10 N.m) is suddenly applied between $1 \mathrm{sec}$ and $2 \mathrm{sec}$, followed by a consign inversion $(-150 \mathrm{rad} / \mathrm{s})$ at $2.5 \mathrm{~s}$. this test has for object the study of controller behaviors in pursuit and in regulation. The test results obtained are shown in Figure 5.

a. It is found that the estimation of speed rotation is almost perfect. The observed speed perfectly tracks the mesurmed speed with almost zero static error. Good sensitivity to load disturbances is observed with a relatively low rejection time, An excellent orientation of the rotor flux on the direct axis is also observed.

b. During the changes of the reference, and especially during the reversal of rotation, the change of the direction of the torque does not degrade the orientation of the fluxes. There is also a perfect continuation of the components of the rotor fluxes estimated at their corresponding real components.

In order to study the influence of parametric variations on the behavior of the vector control without a speed sensor based on sliding mode observer, we introduced a variation of $+50 \%$ of $R_{r}$ in the first test, then a variation of $+50 \%$ of $R_{s}$. We obtained the results as shown in Figures 6 and 7, respectively. It will be noted that at each instant of variation in rotor resistance, the speed observation is almost perfect. An excellent orientation of the rotor flux on the direct axis is also observed. During the changes of the reference, and especially during the reversal of rotation, the change of the direction of the torque does not degrade the orientation of the flow. 


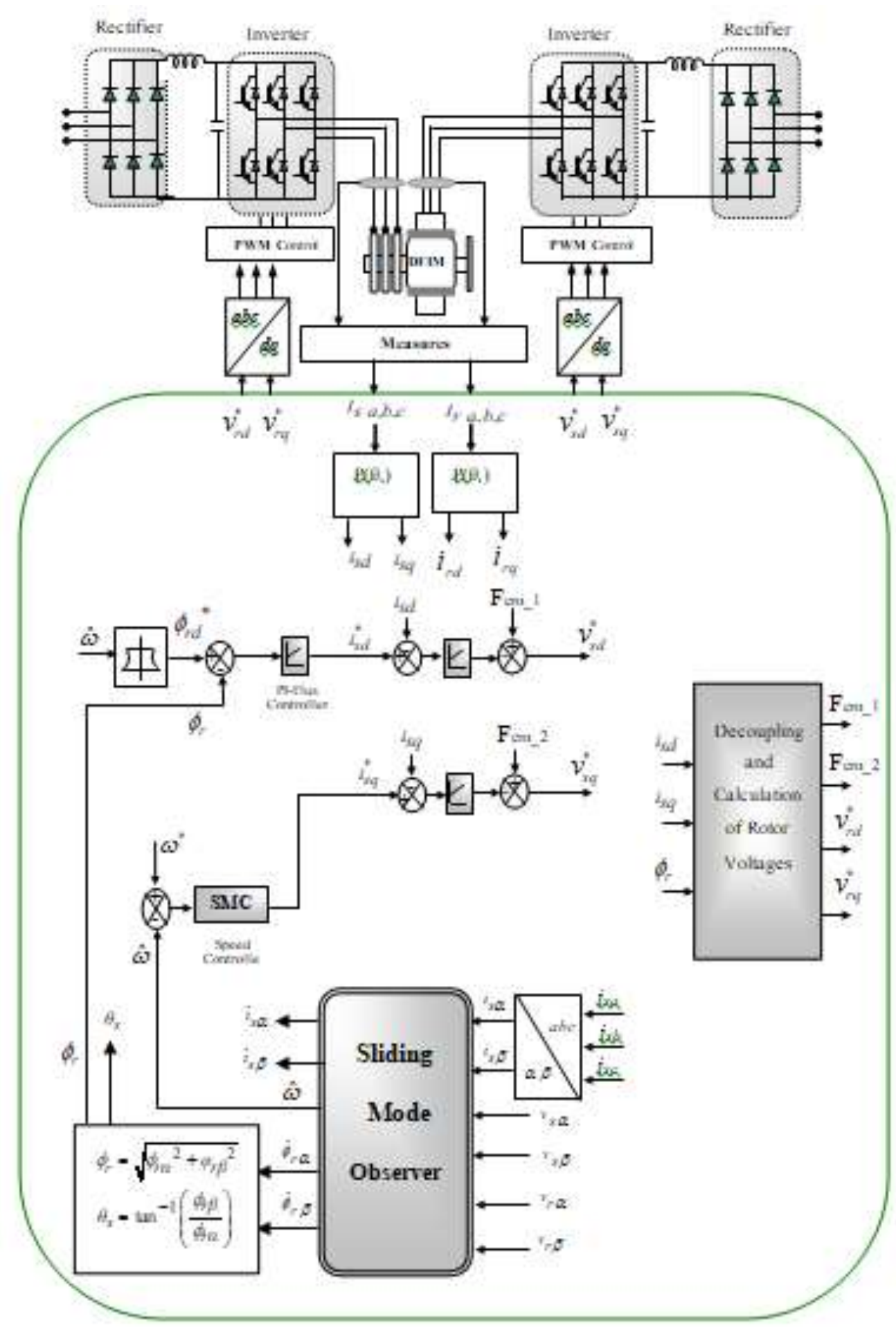

Figure 4. Block diagram of sensorless direct vector control of DFIM using a sliding 

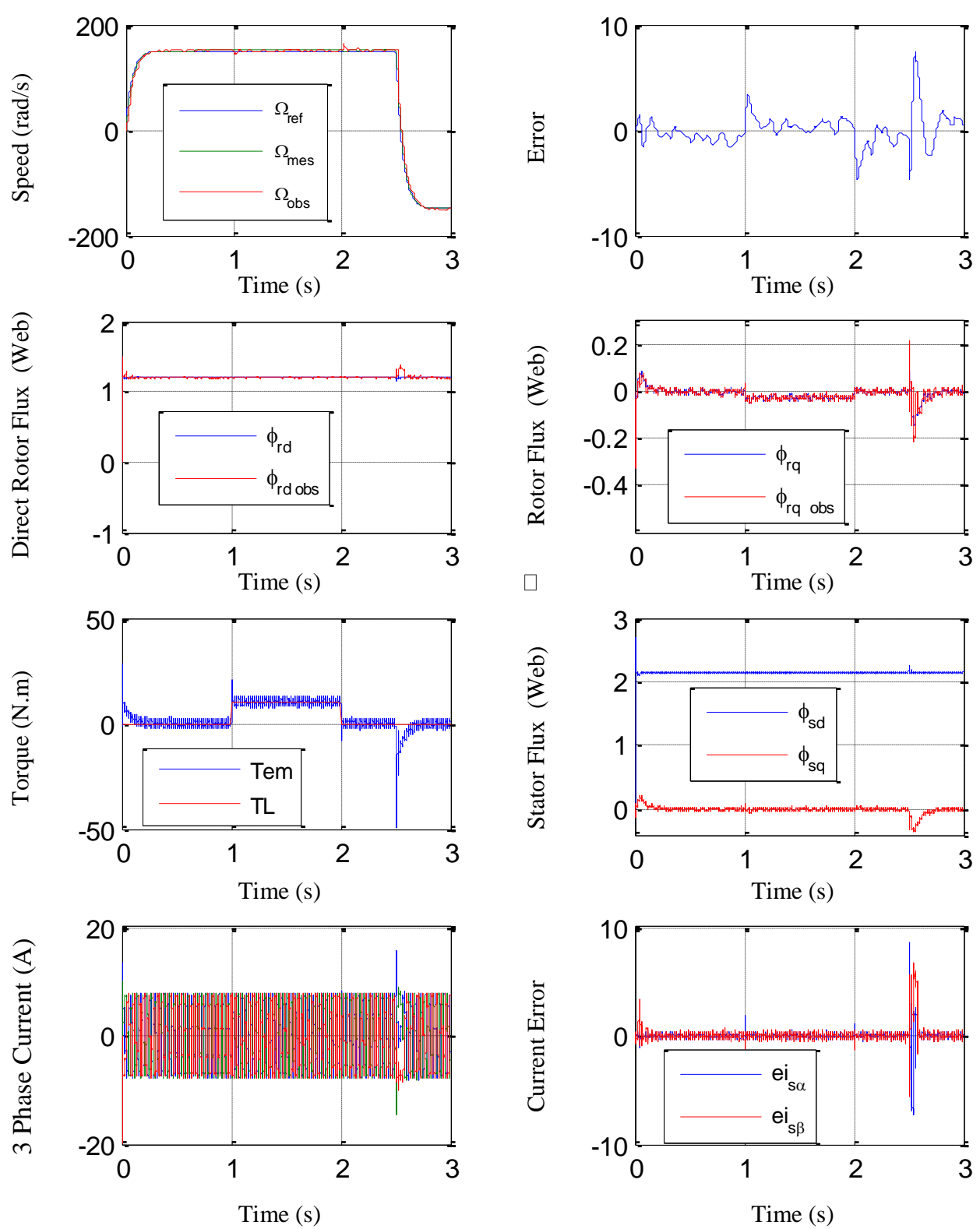

Figure 5. Simulation results of the sensorless speed control using sliding mode observer 

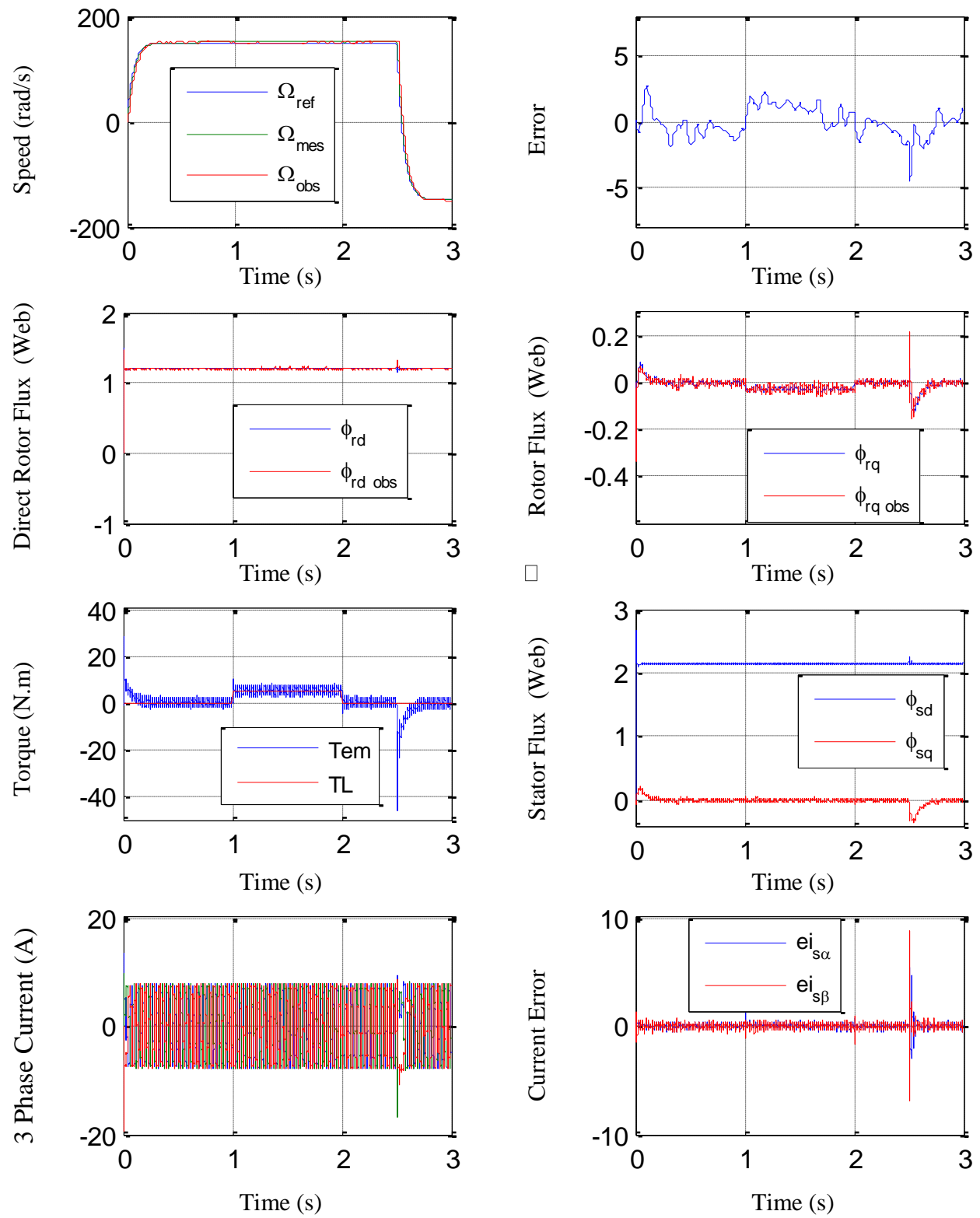

Figure 6. Simulation results of the speed estimation with rotor resistance increased sharply by $50 \%$ from the rated value

For a nominal value of $R_{r}$, the stator resistance $R_{s}$ is increased by+50\% of its nominal value. It is also noticed that this variation does not degrade the orientation of the flux.

Regarding the simulation results obtained, it can be said that our drive without a speed sensor can achieve good performance. 

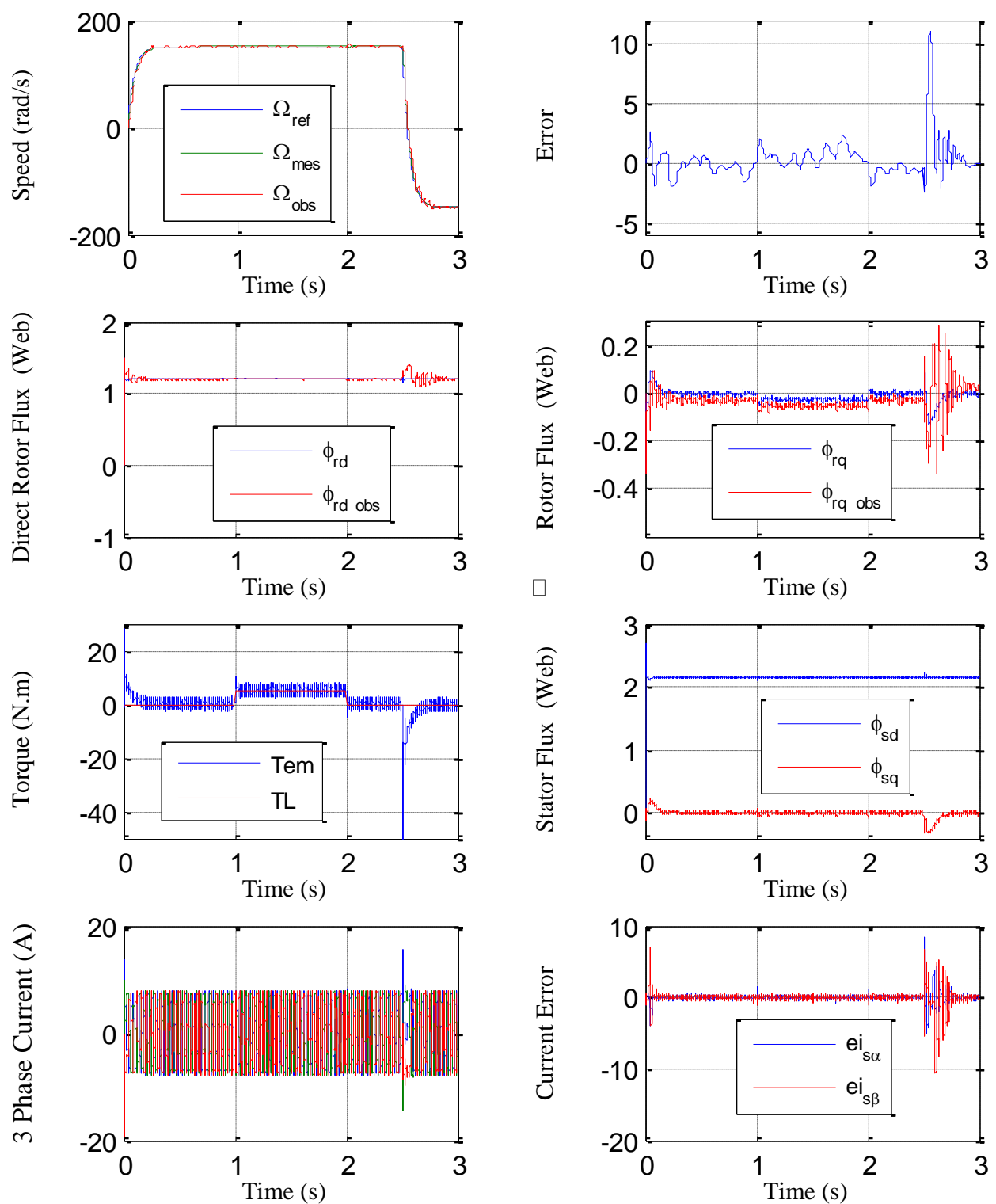

Figure 7. Simulation results of the speed estimation with stator resistance increasad sharply by $50 \%$ from $\mathrm{R}_{\mathrm{sn}}$

To evaluate more robustness of the control, a low speed setpoint of $10 \mathrm{rad} / \mathrm{s}$ was applied. The simulation results are shown in the Figure 8.

It can be seen that the observed speed follows its real value in the presence of the oscillations. An excellent orientation of the rotor flux on the direct axis is also observed. 

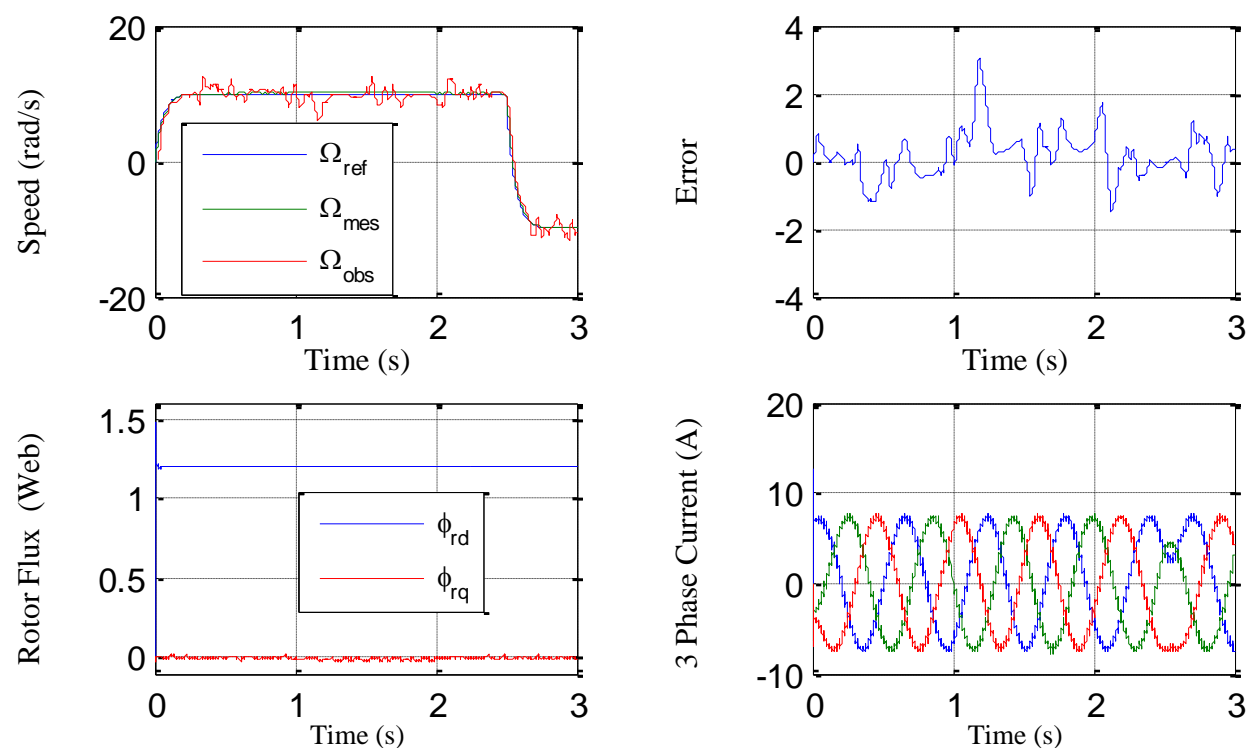

Figure 8. Sensorless speed control using sliding mode observer at low speed

\section{CONCLUSION}

In this paper, it is shown that the use of a sliding mode observer is an effective approach to solving the problem of controlling the DFIM. However, the presentation of the synthesis of our observer of sliding type is very easy. The question of sensitivity of the control system, to the variations of the parameters of the machine, has been widely analyzed. We have focused on the influence of rotor and stator resistances. Extensive tests with different parametric variations show that the proposed observer is more robust and more efficient. Computer simulation results obtained confirm the validity and effectiveness of the proposed control approach at low speed.

\section{REFERENCES}

[1] Abderrahim El Fadili, Fouad Giri, Abdelmounime El Magri, Luc Dugard. "Backstepping controller for Doubly Fed Induction Motor with bi-directional AC/DC/AC converter", CONFIDENTIAL. Limited circulation. For review only 2013.

[2] D. Ben Attous, Y. Bekakra, "Speed Control of a Doubly Fed Induction Motor using Fuzzy Logic Techniques", International Journal on Electrical Engineering and Informatics, Volume 2, Number 3, 2010.

[3] S. Drid, M. Tadjine, M. S. Naît-Saîd, "Robust backstepping vector control for the doubly fed induction motor," The Institution of Engineering and Technology, Control Theory Appl., 2007, 1, (4), pp. 861-868.

[4] S. Drid, M. Tadjine, M.S. Nait-Said, "Nonlinear feedback control and torque optimization of a doubly fed induction motor," Journal of Electrical Engineering, Vol. 56, NO. 3-4, 2005, 57-63.

[5] DJ. Cherifi, Y. Miloud, A. Tahri, "New Fuzzy Luenberger Observer for Performance Evaluation of a Sensorless Induction Motor Drive", International Review of Automatic Control (I.RE.A.CO.), Vol.6, n. 4. July 2013.

[6] Blaschke E. The principle of field orientation as applied to the new trans vector closed-loop control system for rotating field machine. Siemens Review; 1972, Vol. 34 .p. 217-220.

[7] Youcef Bekakra and Djillani Ben Attous, "Comparison between Fuzzy Sliding Mode and Traditional IP Controllers in a Speed Control of a Doubly Fed Induction Motor », Ecti Transactions on Electrical ENG., Electronics, and Communications VOL.10, NO.2 August 2012.

[8] Grzegorz Tarchała, "Sliding Mode Speed Control of an InductionMotor Drive Using Time-varying Switching Line", Power Electronics and Drives, 2(37), No. 1, 2017.

[9] Meziane S, Toufouti R, Benalla H. "MRAS based speed control of sensorless induction motor drives". ICGSTACSE Journal 2007; 7(1):43-50.9.

[10] Yali Zhou, Yongdon Li and Zedong Zheng, "Research of Speed sensorless Vector Control of an Induction Motor Based on Model Reference Adaptive System" 2009 IEEE.

[11] Grzegorz Tarchała, Teresa Orłowska-Kowalska «Sliding Mode Speed Observer for the Induction Motor Drive with Different Sign Function Approximation Forms and Gain Adaptation », Przegląd Elektrotechniczny, ISSN 0033-2097, R. 89 NR 1a/2013.

[12] Arunesh Kumar Singh, and Abhinav Saxena, "A Review on Sensor Less Control of Doubly Fed Induction Machine (DFIM)," Fourth International Conference on Control System and Power Electronics-CSPE. 
[13] Karim Negadi, Abdellah Mansouri, Belkheir Khtemi, "Real Time Implementation of Adaptive Sliding Mode Observer Based Speed Sensorless Vector Control of Induction Motor," Serbian Journal of Electrical Engineering, vol. 7, no. 2, pp. 167-184, November 2010.

[14] Linyun Xiong, Penghan Li, Hao Li and Jie Wang, "Sliding Mode Control of DFIG Wind Turbines with a Fast Exponential Reaching Law", Energies, vol. 10, no. 11, pp. 1-19, 2017.

[15] M.Abid, A.Aissaoui ,H.Bounoua, "Sliding mode application in speed and flux control of an induction machine," Journal of Electrical and Electronics Engineering, vol.6, no.2, pp.197-204, 2006.

[16] J B Alaya, A Khedher, M F Mimouni, "Speed-Sensorless DFIG Wind Drive Based on DTC Using Sliding Mode Rotor Flux Observer", International Journal of Renewable Energy Research, vol.2, no.4, 2012.

[17] F.Mellah, M.Chenafa, A.Bouhenna, A.Mansouri, "Passivity Control with sliding mode observer of induction motor", Przegląd Elektrotechniczny (Electrical Review), ISSN 0033-2097, R. 87 NR 7/2011.

[18] A. F. Payam and M. Jalalifar, "Robust Speed Sensorless Control of Doubly-Fed Induction Machine Based on InputOutput Feedback Linearization Control Using a Sliding-Mode Observer," 2006 International Conference on Power Electronic, Drives and Energy Systems, New Delhi, 2006, pp. 1-5.

[19] Chiheb Ben Regaya, Abderrahmen Zaafouri, Abdelkader Chaari, "A New Sliding Mode Speed Observer of Electric Motor Drive Based on Fuzzy-Logic", Acta Polytechnica Hungarica. vol. 11, no. 3, 2014.

[20] H. Kim, J. Son and J. Lee, "A High-Speed Sliding-Mode Observer for the Sensorless Speed Control of a PMSM," in IEEE Transactions on Industrial Electronics, vol. 58, no. 9, pp. 4069-4077, Sept. 2011.

\section{APPENDIX}

\section{DFIM Parameters}

$1.5 \mathrm{Kw}, 1450 \mathrm{rpm}, 50 \mathrm{~Hz}, \mathrm{R}_{\mathrm{r}}=1.68 \Omega, \mathrm{R}_{\mathrm{s}}=1.75 \Omega$,

$\mathrm{L}_{\mathrm{s}} 295=\mathrm{mH}, \mathrm{L}_{\mathrm{r}}=104 \mathrm{mH}, \mathrm{L}_{\mathrm{m}}=165 \mathrm{mH}, \mathrm{J}=0.01 \mathrm{~kg} \cdot \mathrm{m}^{2}, f=0.0027 \mathrm{~kg} \cdot \mathrm{m}^{2} / \mathrm{s}$ 